Artículos

\title{
LA COVID-19 EN LOS MEDIOS DIGITALES DE LA ARGENTINA. UN ESTUDIO SOBRE LOS DISCURSOS DE CONTROL Y SANCIÓN Y LAS REPRESENTACIONES DE LAS VÍCTIMAS
}

\author{
Arcangeletti Yacante, Carla Antonella
}

Carla Antonella Arcangeletti Yacante aarcangeletti@gmail.com

Universidad Nacional de Cuyo, Argentina

Intersecciones en Comunicación

Universidad Nacional del Centro de la Provincia de Buenos Aires, Argentina

ISSN: $1515-2332$

ISSN-e: 2250-4184

Periodicidad: Anual

vol. 1, núm. 15, 2021

intercom@soc.unicen.edu.ar

Recepción: 15 Diciembre 2020

Aprobación: 08 Febrero 2021

URL: http://portal.amelica.org/ameli,

jatsRepo/216/2161348005/index.html

DOI: https://doi.org/10.51385/ic.v1i15.60

Intersecciones en Comunicación sostiene su compromiso con las políticas de Acceso Abierto a la información científica.

Los autores conservan los derechos de autor y conceden a la revista el derecho de la primera publicación de la obra de forma simultánea bajo la Licencia Licencia Creative Commons Atribución-No ComercialCompartir Igual 4.0 Internacional que permite compartir el trabajo con el reconocimiento de su publicación inicial en Intersecciones en Comunicación. Además, se adhiere a la declaración DORA, y su propuesta mejorar la forma de evaluación científica. La revista cuenta con políticas de preservación de documentos digitales a través del Repositorio Institucional Digital de Acceso Abierto de la Universidad Nacional del Centro de la Provincia de Buenos Aires (https:// www.ridaa.unicen.edu.ar) Intersecciones en Comunicación adhiere a las normas internacionales y prácticas respecto a los aspectos éticos que deben seguirse en el proceso de publicación de artículos originales. En este sentido adhiere a lo que sugiere el Committee on Publications Ethics (https://publicationethics.org/resources/ guidelinesnew/principles-transparency-and-best-practice-scholarlypublishing)

\section{c) (i) (2)}

Esta obra está bajo una Licencia Creative Commons AtribuciónNoComercial-CompartirIgual 4.0 Internacional.
Resumen: El objetivo general de este trabajo es analizar la cobertura mediática de la COVID-19 en la prensa digital argentina. Partiendo de la premisa de que en contextos de pandemia el consumo mediático tiende a elevarse producto de la necesidad de las personas de recibir información de calidad en tiempo real, se pretende identificar: 1) cómo fueron tematizadas las coberturas; 2) si la excepcionalidad de la situación favoreció el despliegue de encuadres de sanción y control, y 3) cómo se construyeron en los medios digitales a las víctimas de la pandemia tras más de 190 días de aislamiento y/o distanciamiento social. En función de los objetivos propuestos, se desarrolló un análisis de contenido cuantitativo sobre las noticias más relevantes sobre COVID-19 publicadas por los diarios digitales Clarín, La Nación, Infobae y Página/12.

Palabras clave: COVID-19, medios online, agenda, riesgo, víctimas, framing.

\begin{abstract}
COVID-19 IN THE ARGENTINIAN DIGITAL MEDIA . A STUDY ON THE PUNITIVE DISCOURSES AND THE REPRESENTATIONS OF THE VICTIMS. The general objective of this work is to analyze the media coverage of COVID-19 in the Argentine digital media. Starting from the hypothesis that in pandemic contexts media consumption tends to rise as a result of people's need to receive quality information in real time, the specific objectives are: 1) identify the particular thematization of the pandemic media coverage; 2 ) analyze if the exceptional nature of the situation favored the deployment of punitive discourses; and 3) determine the patterns which the victims were constructed in digital media after more than 190 days of isolation. Based on the proposed goals, a quantitative content analysis was developed on the most relevant news about COVID-19 published by Clarín, La Nación, Infobae and Página / 12.
\end{abstract}

Keywords: COVID-19, online media, agenda, risk, victims, framing. 


\section{INTRODUCCIÓN}

La pandemia por COVID-19 constituye un proceso, aún abierto, que modificó la vida de millones de personas y en el que los medios de comunicación ocupan un lugar central. Esta preponderancia mediática se debe principalmente a que estos contextos están caracterizados por continuos sucesos extraordinarios que generan una mayor percepción del riesgo. En tales situaciones, el consumo mediático tiende a elevarse producto de la necesidad de las personas de recibir información de calidad en tiempo real (Masip et al., 2020).

Por otro lado, en la pandemia por COVID-19 se identifica a un agente como amenazante para los valores e intereses sociales (Cohen, 2011), por lo que podría ser pensada como activadora de un proceso de pánico moral. En este contexto, el incremento del temor se complementa con una dinámica de estigmatización dirigida a posibles culpables, basadas en percepciones mediáticas y prejuicios sociales preexistentes (McCauley \& Minsky, 2013), escenario que se intensifica en momentos de confinamiento, producto de la imposibilidad de contrastación intersubjetiva de la realidad a la que accedemos a través de diferentes pantallas y plataformas.

En este sentido, este trabajo se propone como objetivo general analizar la cobertura mediática de la COVID-19 en los principales diarios digitales de la Argentina entre el 20 de marzo de 2020, fecha de inicio del Aislamiento Social Preventivo y Obligatorio y hasta el 04 de octubre del mismo año, completándose 28 semanas de observación.

En términos específicos se pretende identificar: 1) cómo fueron tematizadas las coberturas; 2) si la excepcionalidad de la situación favoreció el despliegue de encuadres de sanción y control, y 3) cómo se construyeron en los medios digitales a las víctimas de la pandemia tras más de 190 días de aislamiento y/o distanciamiento social.

\section{CONTEXTO: LA LLEGADA DE LA COVID A LA ARGENTINA}

El primer caso argentino de SARS-CoV-2, una variante novedosa de la familia de los coronavirus, tuvo lugar el 3 de marzo de 2020. La Organización Mundial de la Salud (OMS) declaró pandemia a la COVID-19, enfermedad producida por este patógeno desconocido, el 11 de marzo. En este marco, el Poder Ejecutivo Nacional (PEN) actuó frente a la amenaza de una transmisión acelerada y estableció, a partir de la publicación del Decreto 297/20 en el Boletín Oficial, el Aislamiento Social Preventivo y Obligatorio (ASPO) desde el 20 de marzo.

Desde ese momento los habitantes del territorio argentino ingresaron en una etapa de confinamiento obligatorio sobre el cual el PEN redefinió medidas en relación con la evolución de la pandemia. Así, cada dos semanas el presidente de la Nación, Alberto Fernández, generalmente acompañado por el Gobernador de la Provincia de Buenos Aires, Axel Kicillof; el Jefe de Gobierno de la Ciudad Autónoma de Buenos Aires, Horacio Rodríguez Larreta; y alternativamente por algunos ministros, gobernadores y miembros del comité de expertos que lo asesoran, dio a conocer diferentes medidas sanitarias.

Entre ellas se destacaron el manejo de las fronteras nacionales, de la circulación de personas y mercancías dentro del territorio nacional, el fortalecimiento del sistema público de salud; e iniciativas económicas, como el anuncio del Ingreso Familiar de Emergencia (IFE) para personas con dificultades económicas y el plan de Asistencia de Emergencia para el Trabajo y la Producción (ATP) por el cual el Estado abona parte de los salarios de los trabajadores en relación de dependencia de empresas en crisis que lo soliciten y promueve créditos subvencionados a tasa cero para cuentapropistas. 


\section{MARCO TEÓRICO: AGENDAS PANDÉMICAS Y RIESGOS}

Las epidemias implican problemas de salud que "son percibidos como un peligro no sólo para grupos específicos sino también para poblaciones enteras. Esto produce enormes movilizaciones, tanto de instituciones gubernamentales como de los medios masivos, que le otorgan un lugar central en su cobertura de noticias" (Hallin et al., 2020, p. 2).

Particularmente, en situaciones de crisis como la que generó la pandemia por COVID-19, la demanda informativa por parte de los públicos tiende a aumentar (Farré, 2015), por lo que los medios adquieren mayor centralidad en la definición de las representaciones públicas de la situación. Es decir, en tiempos de pandemia, se refuerza el rol que los medios ocupan en la información y en la conexión de las audiencias con el entorno (Lagos Lira, 2020) producto de un aumento de la demanda de noticias relativas a la enfermedad (Masip et. al. 2020). La incertidumbre generada sobre las características de un nuevo virus incentiva coberturas dramáticas que promueven sentimientos de miedo y respuestas emocionales (Masip et. al, 2020).

Así, las "enfermedades emergentes", entendidas como aquellas que son producidas por un nuevo tipo de patógeno, como el SARS-CoV-2, promueven una cobertura mediática particular que, en términos de Ungar (2008), se desarrolla en tres fases: primero se instala una alarma sobre una amenaza potencial, luego las noticias oscilan entre alarmantes y tranquilizadoras, y, por último, se presentan mensajes contenedores para evitar la instauración del pánico (Hallin et al., 2020). Sin embargo, dentro de cada fase tienen lugar diferentes eventos críticos, que, concebidos como discontinuidades radicales en el acontecer público (Pride, 1995), inciden tanto en la relevancia mediática como en la definición social de los problemas.

Para el estudio de las coberturas informativas de la COVID-19 en la prensa digital argentina este trabajo se nutre de los aportes de la teoría de la Agenda Setting, que en su primer nivel de análisis indaga sobre la transferencia de temas desde la agenda mediática a la agenda pública, prestando especial atención a las propiedades de los temas en relación con los sujetos.

Los temas, definidos como una serie de acontecimientos o tópicos que en el tratamiento informativo son incluidos dentro de una categoría más amplia (Shaw \& McCombs, 1977), según la tipificación de Zucker (1978) pueden clasificarse en "obstrusivos" o experienciales (obstrusive issues) o "no obstrusivos" o no experienciales (unobstrusive issues). Si se tiene en cuenta que aquellos más cercanos a la experiencia directa de los sujetos obstruyen la potencia de las representaciones mediáticas, es posible caracterizar que la pandemia, por tratarse de un tema no experiencial -sobre todo en sus inicios-, exacerbó la centralidad de las representaciones mediáticas sobre la experiencia de los sujetos.

Según la Agenda Setting, las noticias son el resultado de activos procesos de selección, omisión y jerarquización de la información. Estas constituyen el sustrato fundamental de las agendas mediáticas, concebidas como el listado de temas a los que los media otorgan relevancia y, de este modo, contribuyen a incluir en el debate público, (McCombs, 2006). Por relevancia noticiosa se entiende al "nivel de importancia que adquiere un asunto" (Dearing \& Rogers, 1996, p. 8). En términos operacionales, se la estipula a partir de dos dimensiones: la frecuencia de publicación y la jerarquía noticiosa, identificada a partir de diferentes recursos formales y disposicionales de las piezas informativas (Zunino, 2015).

En tanto, en su segundo nivel de análisis, la Agenda Setting incorpora una hipótesis referida a los atributos aplicados por los medios a los diferentes objetos desplegados en sus agendas (McCombs et al., 1997). Definidos como aspectos que acompañan y moldean a las noticias (McCombs \& Valenzuela, 2014), pueden diferenciarse entre sustantivos y afectivos, siendo los primeros relativos a los aspectos sobresalientes de personas, temas u objetos destacados en el tratamiento mediático; mientras que los segundos remiten al tono valorativo resultante de los elementos afectivos desplegados en las noticias, generalmente clasificados en positivos, negativos o indefinidos (Casermeiro de Pereson et al., 2009).

La teoría del Framing, en tanto, como complementaria de la Agenda Setting, también arroja insumos conceptuales para el desarrollo de esta investigación. El Framing es un proceso integral, dinámico e 
interactivo que atraviesa todas las instancias de la comunicación y se relaciona con los modos en que las empresas mediáticas, los comunicadores, las fuentes y la cultura se combinan para producir formas coherentes de comprender el mundo (Koziner, 2013). Según Entman (1993), encuadrar es "seleccionar algunos aspectos de la realidad percibida y hacerlos más relevantes en un texto comunicativo, de modo que se promueva una determinada definición del problema, una interpretación causal, una evaluación moral y/o una recomendación de tratamiento para el asunto descrito" (p.52).

En relación con los conceptos vertidos, este trabajo se propone indagar sobre la composición temática de las agendas de la pandemia y el rol que jugaron en ese marco los discursos punitivos de control y sanción. Asimismo, se procura indagar sobre las configuraciones de las víctimas de la pandemia con el fin de establecer cuáles fueron los patrones de cobertura más persistentes.

\section{METODOLOGÍA}

En función de los objetivos planteados se realizó un análisis de contenido cuantitativo de las piezas informativas más relevantes sobre COVID-19 publicadas en los diarios digitales más leídos del país, a saber, Clarín, La Nación, Infobae y Página 12, en un período que va desde el 20 de marzo del 2020, fecha en la que se decretó el Aislamiento Social Preventivo y Obligatorio (ASPO), hasta el 4 de octubre, completándose 28 semanas de observación.

El análisis de contenido es una técnica de investigación destinada a formular, a partir de ciertos datos manifiestos o latentes, inferencias reproducibles y válidas que pueden aplicarse a su contexto (Krippendorff, 1990). Se trata de una metodología que combina intrínsecamente la recogida y la producción de los datos con su interpretación y análisis.

Para la presente investigación se realizó una recolección de las cinco primeras noticias de cada home en un corte matutino $(9: 00 \mathrm{Hs})$, lo que configuró un universo de 3.885 piezas informativas que constituyeron las unidades de análisis del estudio. El corpus fue codificado en su totalidad.

Los medios seleccionados constituyen las unidades de contexto de la investigación. La Nación fue el primer diario de Buenos Aires en entrar en la web, el 1 de diciembre de 1995. De ese modo, otro de los periódicos centenarios del país, fundado por el ex Presidente Bartolomé Mitre en 1870 (1862-1868), empezaba a desempeñarse en la red (Rost \& Bergero, 2016). El 10 de marzo de 1996 llegó a internet Clarín, el diario papel más leído del país y uno de los de mayor circulación de habla hispana. Fundado por el ex diputado del Partido Socialista Independiente, Roberto Noble, el 28 de agosto de 1945, es la marca insignia del mayor multimedio del país (Becerra, 2015). Página/12 apareció en mayo de 1987. Concebido como un medio de contrainformación, con una agenda diferente a la de los diarios tradicionales, llegó a la web en marzo de 1998. Hoy forma parte del Grupo Octubre, liderado por Víctor Santamaría, secretario general del Sindicato Único de Trabajadores de Edificios de Renta y Horizontal (SUTERH). En tanto, Infobae, creado en el 2002 por el empresario Daniel Hadad, es un nativo digital que al día de la fecha se ubica al tope de lecturas a nivel nacional.

\section{RESULTADOS}

La pandemia por Coronavirus tuvo una relevancia inédita en las coberturas mediáticas. Entendiendo la relevancia en términos de frecuencia y jerarquía (Amadeo, 2008, p. 191), el tema monopolizó las agendas mediáticas.

Si se observa el gráfico $\mathrm{N}^{\circ} 1$ resulta posible advertir que la frecuencia de cobertura de la COVID-19 tuvo fluctuaciones a lo largo del proceso, con una clara tendencia descendente. Así, la determinación del ASPO en la tercera semana de marzo por parte del PEN coincidió con el pico más alto de cobertura, lo que puede explicarse si se concibe a la pandemia como un tema no experiencial (Zucker, 1978) producto de su novedad 
y desarrollo en un contexto de aislamiento social en el que las fuentes de información intersubjetivas se vieron materialmente restringidas. Con el paso de las semanas se suscitaron menores niveles de presencia del tema en las agendas, aunque se registran picos coincidentes con los anuncios presidenciales de extensión o flexibilización de la cuarentena en las distintas regiones del país. En ese sentido, la evolución temática de la agenda estuvo mucho más asociada a la novedad de los anuncios gubernamentales relacionados con la gestión del aislamiento que con la evolución objetiva de la cantidad de casos y fallecimientos producto de la enfermedad.

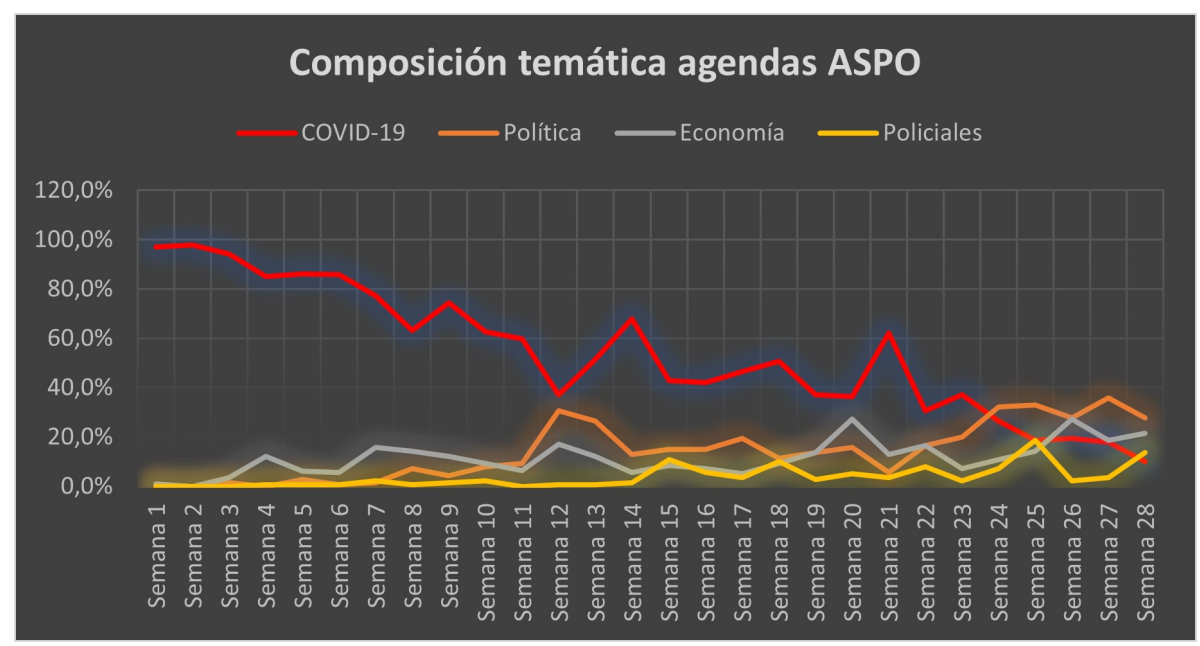

GRÁFICO 1

composición de agendas mediáticas de Clarín, La Nación, Infobae y Página/12. 20 de marzo al 4 de octubre de 2020.

Hacia el final del período analizado, la pandemia alcanzó los niveles más bajos de relevancia. La situación puede ser explicada a partir de la competencia temática que implicó el resurgimiento de otros temas tradicionales de las agendas mediáticas pre-pandémicas. De esta manera, los análisis de la crisis económica que asola al país y al mundo, la polarización política y otros temas "blandos" como las crónicas policiales que instituyen un escenario de "inseguridad" no necesariamente asociados a la pandemia adquieren centralidad y disputan la construcción temática de las agendas.

En ese sentido, también es posible hipotetizar que se dio una transición de la COVID-19 de tema no experiencial a experiencial, producto de la vivencia de la enfermedad en primera persona o por medio de allegados de las audiencias, lo cual desplazó a los medios como únicos constructores de significaciones sobre el asunto.

Como se adelantó, una observación pertinente es la que refleja la disociación entre la evolución de las coberturas mediáticas y el avance de la enfermedad. En efecto, la pandemia afloró como un monotema cuando los casos identificados en el país eran relativamente bajos, mientras que en los días de récords de contagios y fallecimientos el tema adquirió escasa visibilidad.

Por otro lado, dentro de las coberturas mediáticas referidas a la COVID-19 han pujado diversos enfoques temáticos. Si bien la dicotomía entre "salud” y "economía” para abordar la problemática fue una constante, surgió además un encuadre de "sanción y control” asociado al aislamiento que, aunque menor en términos de visibilidad, resulta determinante para el análisis de la construcción mediática del riesgo.

El gráfico $\mathrm{N}^{\circ} 2$ da cuenta del modo en que un enfoque sanitario, vinculado con la evolución de los casos, información sobre las muertes, avances de vacunas y terapias, y medidas de prevención dispuestas por el gobierno nacional y los provinciales, confrontó con un enfoque político-económico que encuadró la situación desde las consecuencias económicas de la pandemia y el aislamiento. 


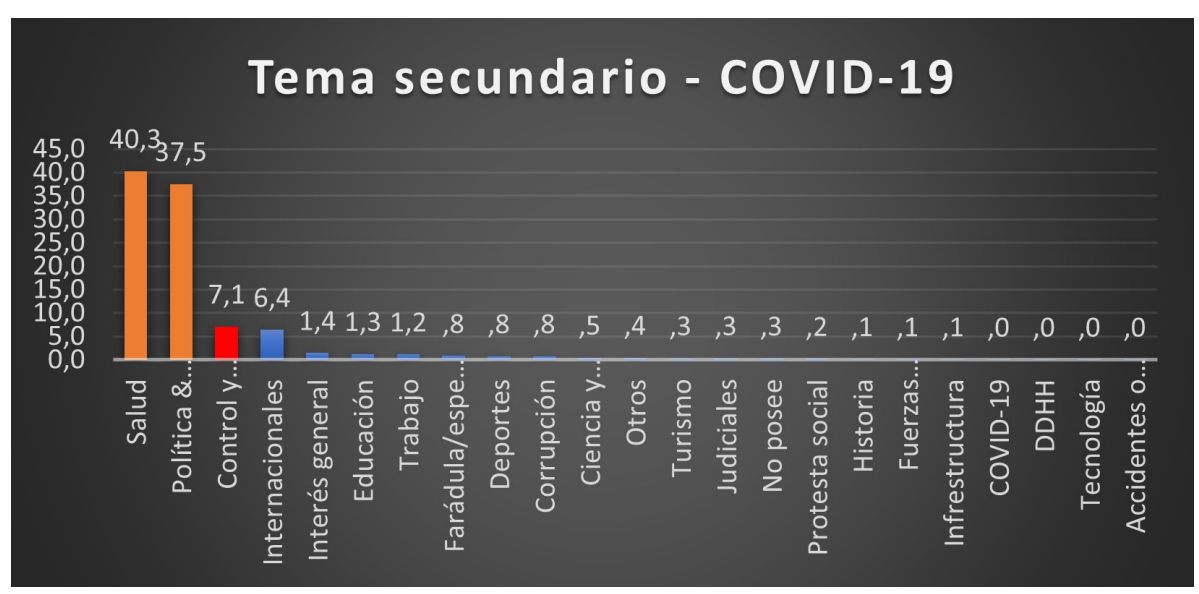

GRÁFICO 2

frecuencia de tópicos secundarios asociados a COVID-19. Clarín, La Nación, Infobae y Página/12. 20 de marzo al 4 de octubre de 2020

En tanto, en tercer lugar se puso en juego un encuadre, subyacente a los dos dominantes, que se construyó a partir de discursos de control y sanción, asociados sobre todo al cumplimiento o incumplimiento de las normas de aislamiento. Este enfoque fue alentado por discursos gubernamentales que pusieron el foco en la necesidad del cumplimiento de las normas dispuestas a partir de una motivación sanitaria y solidaria, pero que a la vez explicitaron una serie de sanciones graves para las violaciones de las disposiciones, al tiempo que alentaron la denuncia ciudadana a los incumplidores.

Las oscilaciones que presentó la presencia mediática de este encuadre a lo largo del ASPO manifiesta sus picos más altos asociados a los anuncios de endurecimiento de la cuarentena. En los días posteriores a cada decisión gubernamental los medios pusieron el foco tanto en la prescripción de las actividades habilitadas y prohibidas, cuanto en la visibilización de los dispositivos coercitivos de control y sanción, como operativos policiales en la vía pública justificados en un estado de excepción que habilitó al Estado a restringir la circulación y reunión bajo amenaza de sanción.

En tanto, si se analizan las valoraciones mediáticas sobre los diversos agentes públicos, las fuerzas de seguridad obtienen el segundo lugar en reconocimiento, con un $41,5 \%$ de valoración positiva y un $26 \%$ de valoración negativa, siendo únicamente superado por el Ministerio de Salud que, pese a un declive hacia el final del período, acapara un $46,5 \%$ de tono valorativo positivo y un $18 \%$ de evaluación negativa.

Ahora bien, otro de los propósitos centrales de este trabajo fue analizar cuál fue el estereotipo de actor que los medios construyeron simbólicamente como víctima de la pandemia en cada etapa del período. Este estudio parte de la distinción entre dos categorías de víctimas: directas e indirectas. Las primeras están conformadas por aquellas personas que efectivamente padecieron la enfermedad. Las segundas, en cambio, son aquellas que sufrieron otras consecuencias, sobre todo económicas, asociadas al aislamiento.

En el comienzo del período las víctimas directas mediatizadas eran representativas de la clase alta o mediaalta que retornaba al país luego de vacacionar en el exterior. Las coberturas mediáticas construyeron estas historias a partir de un encuadre de interés humano (Semetko \& Valkenburg, 2000) basado en un sesgo de personalización (Bennett, 1991) establecido con tono anecdótico. Pero, como es posible advertir en el gráfico $\mathrm{N}^{\circ} 3$, a partir de la semana 5 de ASPO, promediando el mes de abril, las coberturas se volcaron abruptamente a la representación de las clases bajas como las principales afectadas por la enfermedad, producto de la transmisión masiva del virus en los barrios marginalizados del Área Metropolitana de Buenos Aires. En este punto, el abordaje incluyó ribetes morbosos y revictimizadores basados en la presentación de estos sectores sociales como potencialmente peligrosos para el resto de los ciudadanos. 


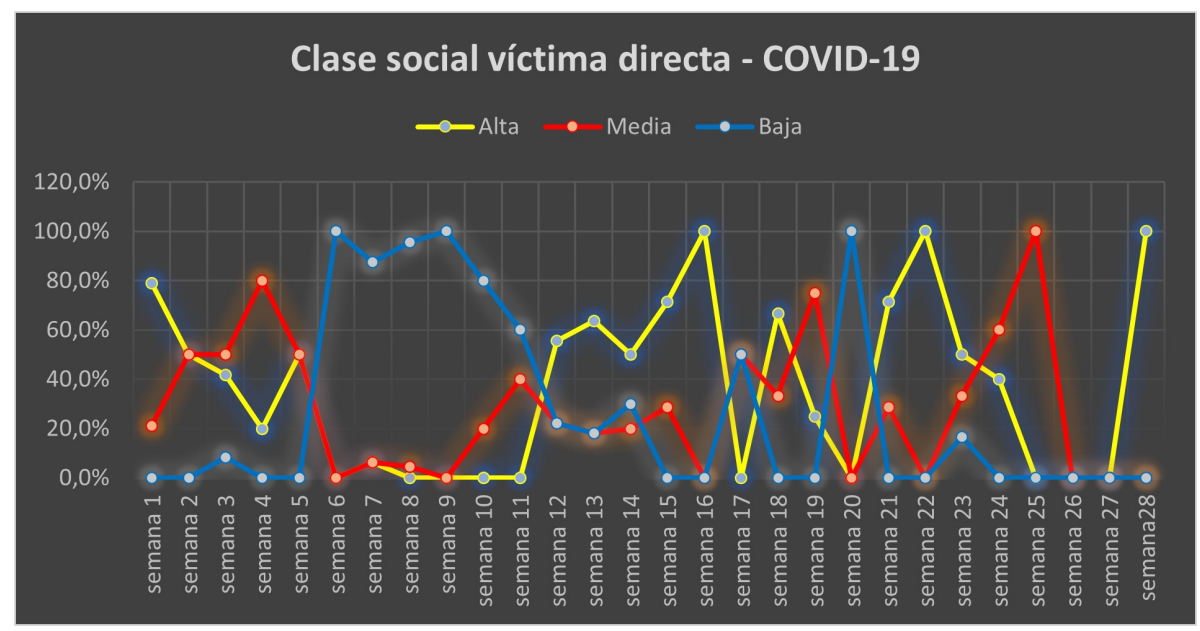

GRÁFICO 3

Clase social de las víctimas directas de COVID-19. Clarín, La Nación, Infobae y Página/12. 20 de marzo al 4 de octubre de 2020

Sin embargo, en poco tiempo los sectores populares volvieron a ser invisibilizados en virtud de un reposicionamiento de la clases medias y altas como víctimas de la pandemia. Tal situación fue producto de que la masificación de la transmisión comunitaria del virus reorientó la atención mediática a ciertos casos de enfermos puntuales que resonaron en las agendas por tratarse de personajes destacados de los mundos del deporte, el arte, la farándula o la política.

La evolución de la victimización directa construida por los medios digitales más importantes del país, en suma, permite establecer una disparidad en la representación de las víctimas directas de la enfermedad. Mientras que el tono distendido y anecdótico se utilizó para contar las experiencias del padecimiento de las clases medias y altas, la invasión a la privacidad, el morbo y la revictimización dominaron el tratamiento informativo de los padecimientos de los sectores populares. Finalmente, la invisibilización mediática de estos sectores hacia el final del período da cuenta de una sobrerrepresentación de los sectores medios como principales afectados por la enfermedad.

En tanto, con la construcción mediática de las víctimas indirectas sucedió algo similar. Tal como surge de los datos expresados en el gráfico $\mathrm{N}^{\circ} 4$, la inicial preocupación por cómo iban a sobrellevar materialmente las clases bajas el ASPO quedó rápidamente eclipsada por una sobrerrepresentación de los padecimientos de los sectores medios y altos, a los que se instituyó como víctimas totalizantes de la pandemia.

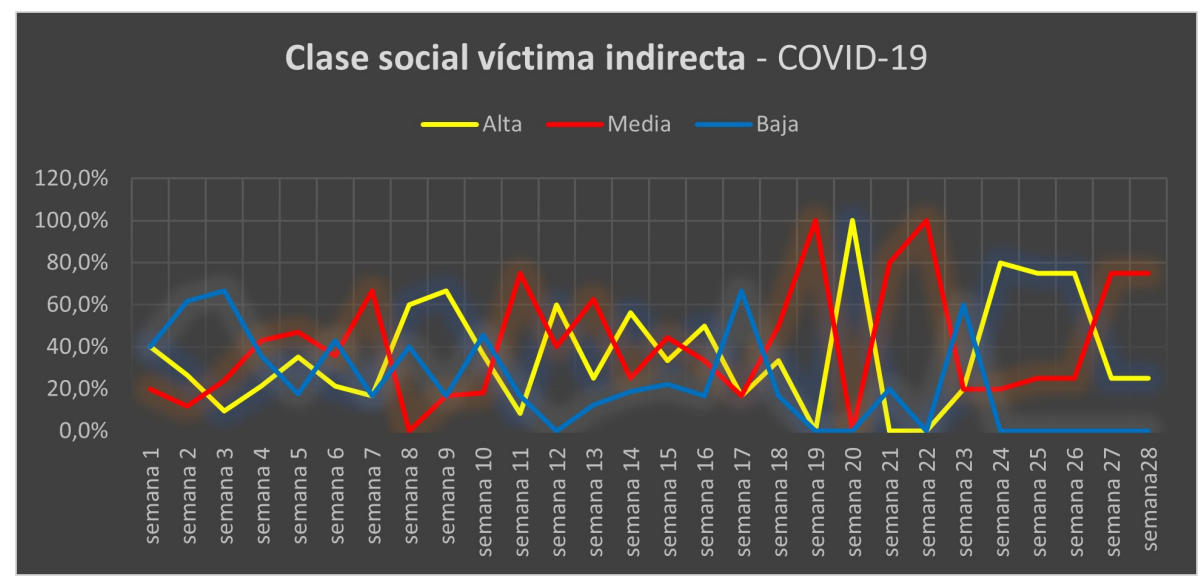

GRÁFICO 4

Clase social de las víctimas indirectas de COVID-19. Clarín, La Nación, Infobae y Página/12. 20 de marzo al 4 de octubre de 2020 
Tal como sucedió con el caso de la victimización directa, los principales afectados por las consecuencias económicas de la COVID-19 y el aislamiento fueron los sectores medios e incluso altos. La afirmación, que se basa sobre todo en la constatación de una alta frecuencia de noticias sobre las dificultades de comerciantes, profesionales, cuentapropistas e incluso de grandes empresas para afrontar las consecuencias económicas de la crisis sanitaria, tendió al mismo tiempo a invisibilizar las dificultades económicas de las clases populares en este particular contexto.

\section{CONCLUSIONES}

La irrupción del SARS-CoV 2 constituyó un evento extraordinario que agudizó la demanda de información de las audiencias brindándole un lugar central a los medios de comunicación.

Los resultados de este estudio permiten observar cómo la pandemia dominó las agendas mediáticas a lo largo del período analizado. En este sentido, se puede advertir que cinco de cada diez noticias publicadas tuvieron como tópico principal a la COVID-19.

Ahora bien, esta supremacía no fue permanente, sino que ha tenido oscilaciones con el transcurso del tiempo. Mientras que el coronavirus se constituía casi como el único tema a fines de marzo y principios de abril, tendió a descender hacia el final del recorte propuesto a valores ínfimos de cobertura. En ese sentido es posible concluir que la noticiabilidad de la pandemia estuvo mucho más asociada a las decisiones políticas de gestión de la crisis que a la evolución objetiva de los contagios.

Por su parte, los encuadres de la pandemia también han mostrado variaciones. Los datos obtenidos reflejan una clara dicotomía propuesta para analizar el escenario que comprende, en un polo, a las políticas y perspectivas sanitarias alentadas por los agentes oficiales y, en el otro, una mirada crítica hacia el aislamiento y sus repercusiones sobre el acontecer económico.

Sin embargo, en este punto resulta pertinente destacar la emergencia de un tercer encuadre de "sanción y control" que, pese resultar marginal en relación con el debate "salud versus economía", no deja de ser significativo como enfoque subyacente. En este sentido, en las agendas mediáticas analizadas una de cada diez piezas informativas promueve soluciones punitivas, congruentes también con un discurso oficial que alentó la obligación de cumplir con las normas dispuestas bajo amenaza de sanción. En este sentido, no es sorprendente que las fuerzas de seguridad hayan resultado uno de los agentes públicos mejor valorados por los medios de comunicación.

Por último, este trabajo se propuso analizar la construcción mediática de las víctimas de la pandemia. Los resultados evidencian que, si bien hubo momentos específicos en los que la atención se detuvo sobre los sectores populares, en general han sido los sectores medios y altos quienes han encontrado en las coberturas mediáticas una sobrerrepresentación como víctimas directas e indirectas de la pandemia.

El presente trabajo constituye un estudio de caso sobre un evento de dimensiones superlativas que, al término de este artículo, se encuentra en plena evolución. Los datos aportados, por lo tanto, resultan preliminares. Sin embargo, permiten trazar algunas tendencias que necesariamente requerirán ser contrastadas luego del cierre del proceso y el paso del tiempo. La relevancia de la pandemia, su tematización en los medios y la construcción de las víctimas en un escenario de riesgos y miedos son sólo algunas dimensiones de análisis posibles sobre un objeto que requiere de enfoques complejos y multidimensionales. Los hallazgos provisorios que se proponen constituyen tan sólo un punto de partida que invita a futuros abordajes más amplios y abarcativos.

\section{REFERENCIAS}

Amadeo, B. (2008). Framing: Modelo para armar. En M. T. Baquerín de Riccitelli (Ed.), Los medios caliados o enemigos del público? (1.a ed., Número 1974, pp. 183-281). EDUCA. 
Bennett, W. L. (1991). News: The Politics of Illusion, Ninth Edition (1.a ed.). Longman.

Casermeiro de Pereson, A., de la Torre, L., \& Téramo, M. T. (2009). Buenos Aires elige presidente. Un estudio en el marco de la teoría de la agenda setting (1.a ed., Número 2009). EDUCA.

Cohen, S. (2011). Folk Devils and Moral Panics. En Contemporary Review (4.a ed., Vol. 282, Número 1649). Taylor \& Francis e-Library.

Dearing, J., \& Rogers, E. (1996). Agenda Setting (1.a ed.). Sage Publications.

Entman, R. M. (2003). Framing: Toward Clarification of a Fractured Paradigm. Journal of Communication, 43(4), 51-58. https://doi.org/10.1111/j.1460-2466.1993.tb01304.x

Entman, R. M. (1993). Framing: Toward clarification of a fracture paradigm. Journal of Communication, 43(4), 51-58.

Farré Coma, J. (2015). Comunicación de riesgo y espirales del miedo. Comunicación y Sociedad, 0(3), 95-119. http:// www.comunicacionysociedad.cucsh.udg.mx/index.php/comsoc/article/view/4209/3969

Hallin, D. C., Briggs, C. L., Mantini-Briggs, C., Spinelli, H., \& Sy, A. (2020). Mediatización de las epidemias: la cobertura sobre la pandemia de la gripe A (H1N1) de 2009 en Argentina, Estados Unidos y Venezuela. Comunicación y Sociedad, e7207, 1-24. https://doi.org/10.32870/cys.v2020.7207

Koziner, N. (2013). Antecedentes y fundamentos de la teoría del framing en comunicación. Austral Comunicación, 2(1), 1-25. http://webtest.austral.edu.ar/ojs/index.php/australcomunicacion/article/view/53

Lagos Lira, C. (2020). Pandiorismo. O periodismo en tiempos de pandemia. Revista Anales, 17, 335-355.

Masip, P., Aran-Ramspott, S., Ruiz-Caballero, C., Suau, J., Almenar, E., \& Puertas-Graell, D. (2020). Consumo informativo y cobertura mediática durante el confinamiento por el Covid-19: sobreinformación, sesgo ideológico y sensacionalismo. El profesional de la información, 29(3), 1-12. https://doi.org/10.3145/epi.2020.may.12

McCauley, M., \& Minsky, S. (2013). The H1N1 pandemic: Media frames, stigmatization and coping. BMC Public Health, 13(1), 1-16. https://doi.org/10.1186/1471-2458-13-1116

McCombs, M. (2006). Estableciendo la agenda. El impacto de los medios en la opinión pública y el conocimiento (1.a ed.). Paidós Ibérica.

McCombs, M., Llamas, J. P., López Escobar, E., \& Rey Lennon, F. (1997). Candidate Images in Spanish Elections: Second-level Agenda-Setting Effect. Journalism and Mass Communication Quaterly, 74(4), 703-717.

McCombs, M., \& Valenzuela, S. (2014). Agenda-Setting Theory: The Frontier Research Questions (Número September 2014). https://doi.org/DOI: 10.1093/oxfordhb/9780199793471.013.48

Pride, R. A. (1995). How Activists and Media Frame Social Problems: Critical Events Versus Performance Trends for Schools. Political Communication, 12(1), 5-26.

Semetko, H. A., \& Valkenburg, P. M. (2000). Framing European politics: a content analysis of press and television news. Journal of Communication, 50(2), 93-109. https://doi.org/10.1111/j.1460-2466.2000.tb02843.x

Shaw, D., \& McCombs, M. (1977). The Emergence of American Political Issues. The Agenda Setting Function of the Press (1.a ed.). West Publishing Company.

Zucker, H. G. (1978). The Variable Nature of News Media Influence. En B. D. Rubin (Ed.), Communication Yearbook 2 (pp. 225-245). Transaction Publishers.

Zunino, E. (2015). La relevancia de las noticias en la prensa gráfica. Una reflexión teórico metodológica a partir del análisis del conflicto entre las corporaciones agrarias y el gobierno argentino, en 2008. Comunicación y Sociedad, 25(enero-julio 2016), 1-32. 